\title{
Preparation of W-Containing Mesoporous Silica Thin Films and Their Surface Hydrophilic Properties*
}

\author{
Yu Horiuchi, Takashi Kamegawa, Kohsuke Mori, and Hiromi Yamashita ${ }^{\dagger}$ \\ Division of Materials and Manufacturing Science, \\ Graduate School of Engineering, Osaka University, \\ 2-1, Yamada-oka, Suita, Osaka 565-0871, Japan \\ Norikazu Nishiyama \\ Department of Materials Engineering Science, Graduate School of Engineering Science, \\ Osaka University, 1-3, Machikaneyama-cho, Toyonaka, Osaka 565-0871, Japan \\ (Received 5 November 2008; Accepted 19 January 2009; Published 7 March 2009)
}

\begin{abstract}
The present paper deals with surface superhydrophilic properties of mesoporous silica thin films containing tungsten oxide species (W-MSTFs) prepared by a sol-gel/spin coating method. The local structure around tungsten oxide species as well as photoinduced surface properties have been investigated by XRD, UV-vis, and water contact angle (CA) measurements. These investigations showed that tungsten oxide species are tetrahedrally-coordinated and exist in isolated states. The water CA measurements also demonstrated that W-MSTFs exhibited the high surface hydrophilic property without the UV-light irradiation. It was also found that the UV-light irradiation led to the decrease in the CA of W-MSTFs, indicating that W-MSTFs showed photoinduced superhydrophilicity. Furthermore, very low CA below $10^{\circ}$ was maintained for about three months in the dark conditions after the UV-light irradiation. [DOI: 10.1380/ejssnt.2009.141]
\end{abstract}

Keywords: Wetting; Tungsten oxide; Silicon oxides; Superhydrophilicity; Mesoporous silica; Thin film

\section{INTRODUCTION}

Fabrication of nanoscaled materials has become a key technology for developing new fields. In particular, nanostructured materials having well-ordered porous structure have attracted much attention in the viewpoint of their applications for the host materials, adsorbent, and electronic devices. As a promising candidate for these materials, powdered zeolite and mesoporous silica were extensively investigated and showed fascinating properties due to their large surface area and framework topology [1-4]. Among them, the porous silica materials containing highly dispersed transition metal oxide species within their frameworks, which were called as single-site photocatalysts, show peculiar properties as compared to bulk metal oxides in their powder form [5-8]. For the further application, utilization in the form of thin films is also very important [9-11].

Recently, our group has developed the Ti-containing mesoporous silica thin film, which is containing tetrahedrally-coordinated titanium oxide species (TiMSTF). This film exhibited highly hydrophilic property without the UV-light irradiation, and also showed photoinduced superhydrophilicity under the UV-light irradiation $[12,13]$. The addition of other transition metal oxide species within the framework of mesoporous silica thin films can be expected to design of highly functional materials.

In this study, W-containing mesoporous silica thin films

* This paper was presented at International Symposium on Surface Science and Nanotechnology (ISSS-5), Waseda University, Japan, 9-13 November, 2008.

†Corresponding author: yamashita@mat.eng.osaka-u.ac.jp
(W-MSTFs) were prepared and their local structures around tungsten oxide species were characterized using spectroscopic measurements. Additionally, their surface hydrophilic properties were also investigated by water contact angle measurements before and after the UV-light irradiation.

\section{EXPERIMENTAL}

\section{A. Materials}

Tetraethyl orthosilicate $\left(\left(\mathrm{C}_{2} \mathrm{H}_{5} \mathrm{O}\right)_{4} \mathrm{Si} ; \quad\right.$ TEOS $)$, ammonium tungstate para pentahydrate $\left(\left(\mathrm{NH}_{4}\right)_{10} \mathrm{~W}_{12} \mathrm{O}_{41} \cdot 5 \mathrm{H}_{2} \mathrm{O}\right), 5 \mathrm{~N}$ hydrochloric acid, and ethanol were obtained from Wako Pure Chemical Ind., Ltd. Polyethylene glycol dodecyl ether as structure directing agent $(\mathrm{SDA})\left(\mathrm{C}_{12} \mathrm{H}_{25} \mathrm{O}\left(\mathrm{CH}_{2} \mathrm{CH}_{2} \mathrm{O}\right) \mathrm{H}\right)$ described as Brij ${ }^{\circledR} 30$ was obtained from Sigma-Aldrich Co. All chemicals were used as received.

\section{B. Synthesis}

Pure mesoporous silica thin film (MSTF) and Wcontaining mesoporous silica thin films (W-MSTFs) were prepared on quartz plates $(10 \times 10 \times 1 \mathrm{~mm})$ using a solgel/spin coating method in the presence of SDA. A mixture of TEOS, aqueous solution of $\left(\mathrm{NH}_{4}\right)_{10} \mathrm{~W}_{12} \mathrm{O}_{41} \cdot 5 \mathrm{H}_{2} \mathrm{O}$, Brij ${ }^{\circledR} 30, \mathrm{HCl}$, and $\mathrm{EtOH}$ with the following molar ratios: $\mathrm{Si}+\mathrm{W}:$ Brij ${ }^{\circledR} 30: \mathrm{HCl}: \mathrm{EtOH}=1.5: 0.18: 0.15: 10(\mathrm{Si}: \mathrm{W}=$ $100: x ; x=0,0.5,1$, and 2 ) was stirred at $293 \mathrm{~K}$ for 15 min. Thus obtained sol was dripped onto quartz plate, spread evenly, and coated at a spinning rate of 4,000 rpm for $1 \mathrm{~min}$ followed by calcination in air at $523 \mathrm{~K}$ for $5 \mathrm{~h}$ at a heating rate of $1^{\circ} \mathrm{C} / \mathrm{min}$. 


\section{OSAKA UNIVERSITY \\ (a) \\ (b) \\ (c) \\ (d)}

FIG. 1: Photographic images of quartz plate (a) and WMSTFs with different tungsten concentration $(\mathrm{W} / \mathrm{Si}=0.005$ (b), 0.01 (c), and $0.02(\mathrm{~d})$ ).

\section{Characterization}

Standard $\theta-2 \theta$ X-ray diffraction (XRD) data were recorded on a Rigaku Mini-flex using $\mathrm{Cu} \mathrm{K} \alpha$ radiation of wavelength $1.5418 \AA$. The diffuse reflectance absorption spectra were monitored by a Shimadzu UV-2450 spectrometer at $293 \mathrm{~K}$. The reference sample was $\mathrm{BaSO}_{4}$, and the absorption spectra were obtained by using KubelkaMunk function. The water-repellency of the film surface was checked by the contact angle of pure water $(3 \mu \mathrm{l})$ using a contact angle meter DropMaster 300 of Kyowa Interface Science CO., LTD. A $10 \mathrm{~mW}$ He-Ne laser, a bandpass filter, a high-resolution $(2000 \times 1312$ pixels $)$ digital camera with a remote controller, a video and an image automatic transmission and processing system were used for the measurement. Because the band-pass filter can cut all other wavelengths except for the laser beam (632 $\mathrm{nm})$, the reflected light can be removed and high definition drop profiles can be obtained. The film surface was irradiated with UV-light using a $200 \mathrm{~W}$ mercury xenon lamp of SAN-EI ELECTRIC CO., LTD at $60 \mathrm{~mW} / \mathrm{cm}^{2}$ for $2 \mathrm{~h}$.

\section{RESULTS AND DISCUSSIONS}

The synthesized W-containing mesoporous silica thin films (W-MSTFs) and pure mesoporous silica thin film (MSTF) showed good adhesion to quartz plates. These thin films were colorless transparent like original quartz plate, as shown in Fig. 1. The scanning electron microscopy (SEM) image provides information about film thickness and surface conditions. Figure 2 shows the cross-section SEM image of the W-MSTF with the W/Si ratio of 0.01 . The mean film thickness of this thin film was determined to be about 1.0-1.5 $\mu \mathrm{m}$. Moreover, any cracks and large particles could not be observed from this image.

Figure 3 shows low angle X-ray diffraction (XRD) patterns of W-MSTFs with different tungsten concentration $(\mathrm{W} / \mathrm{Si}=0.005,0.01$, and 0.02). All thin films exhibited a single diffraction peak at around $2 \theta=4^{\circ}$ associated with the $\mathrm{d}_{100}$ spacing, indicating the presence of the mesoporous structure. On the contrary, in the higher angle region, other characteristic peaks assigned to tungsten oxide crystal could not be observed (data not shown). This result suggests that tungsten oxide species within the mesoporous silica framework are present in highly dispersed states.

The diffuse reflectance UV-visible absorption spectra

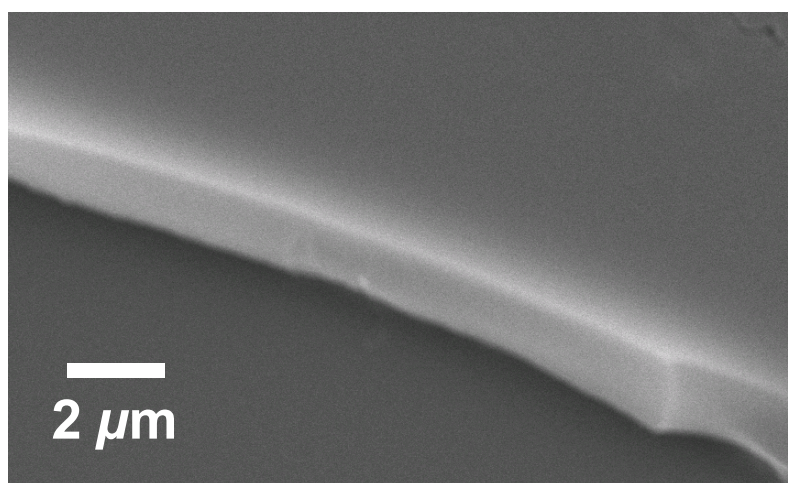

FIG. 2: Cross-section SEM image of W-MSTF (W/Si = 0.01).

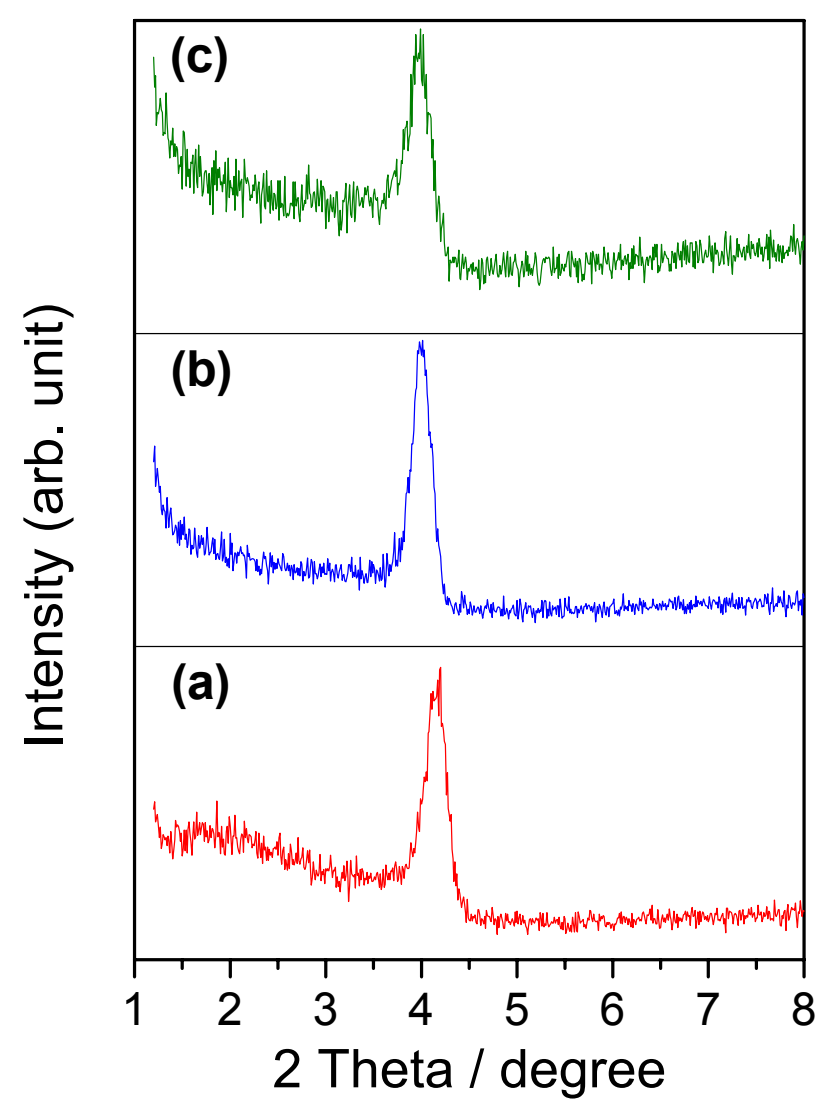

FIG. 3: Low angle XRD patterns of W-MSTFs with different tungsten concentration $(\mathrm{W} / \mathrm{Si}=0.005(\mathrm{a}), 0.01(\mathrm{~b})$, and 0.02 (c)).

of W-MSTFs with different tungsten concentration were shown in Fig. 4. Absorption bands of these thin films were observed in the wavelength region of 250-300 nm. Peak intensities of these bands were increased with increasing the amounts of incorporated tungsten oxide species. These absorption bands in the UV-wavelength region were attributed to the ligand-to-metal charge-transfer (LMCT) band from $\mathrm{O}^{2-}$ to $\mathrm{W}^{6+}$ ions in tetrahedrallycoordinated tungsten oxide species. It was revealed that tungsten oxide species within W-MSTFs exist in isolated tetrahedrally-coordinated states without the formation of aggregated octahedrally-coordinated species $[14,15]$. 


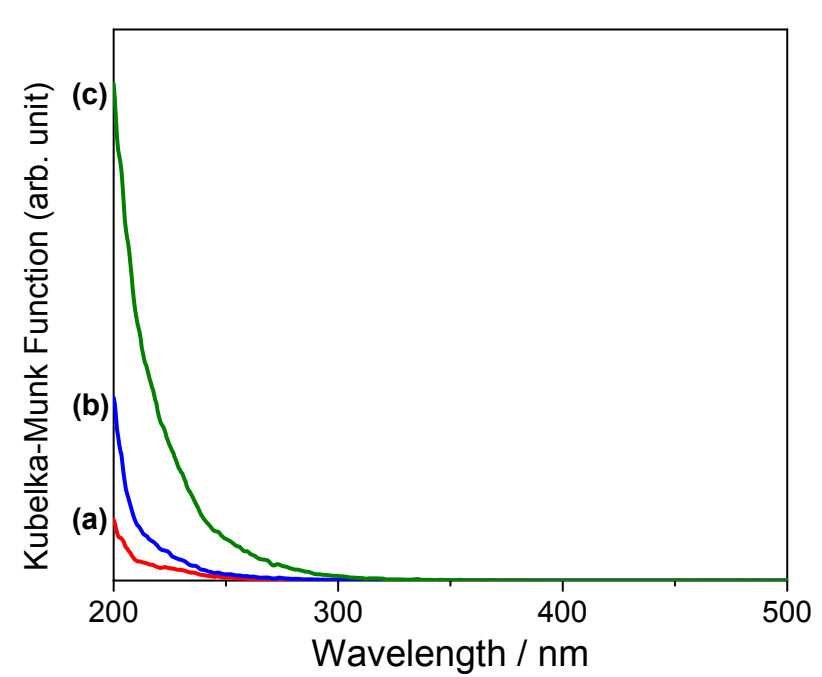

FIG. 4: UV-vis absorption spectra of W-MSTFs with different tungsten concentration $(\mathrm{W} / \mathrm{Si}=0.005(\mathrm{a}), 0.01(\mathrm{~b})$, and 0.02 (c)).

On the other hands, pure MSTF without tungsten oxide species exhibited almost no absorption bands in the UV-visible region (data not shown).

To obtain the information about surface hydrophilic properties of synthesized thin films, contact angle (CA) measurements of water droplets were carried out. Figures 5 and 6 show the $\mathrm{CA}$ and photographic images of water droplets on titanium dioxide $\left(\mathrm{TiO}_{2}\right)$ thin film prepared by the magnetron sputtering deposition method as reference sample, pure MSTF, and W-MSTFs with different tungsten concentration before and after the UV-light irradiation. The $\mathrm{CA}$ on pure MSTF before the UV-light irradiation is about $30^{\circ}$ and much smaller than that on $\mathrm{TiO}_{2}$ thin film (ca. $80^{\circ}$ ). These results suggest that the mesoporous structure can enhance the surface hydrophilic property due to the capillary condensation. However, almost no change of the CA on pure MSTF was observed after the UV-light irradiation, indicating that pure MSTF can not show the photoinduced superhydrophilicity. On the contrary, the CA of all W-MSTFs were very small (ca. $3^{\circ}$ ) even before the UV-light irradiation. These high surface hydrophilic properties without the UV-light irradiation are valuable for practical usages. Moreover, after the UV-light irradiation, the CA on these thin films became extremely small, suggesting that W-MSTFs show the photoinduced superhydrophilicity. It was also confirmed that the W-MSTF has highly hydrophilic properties from the photographic images of the water droplets on these thin films (Fig. 6). These observations pointed that the charge separation induced by the formation of charge transfer excited states of tetrahedrally-coordinated tungsten oxide species formed under the UV-light irradiation play a crucial role in the photoinduced superhydrophilicity, which may modify the surface local polarity related to the appearance of the superhydrophilic property.

The durability of the W-MSTF (W/Si $=0.01$ ) was also investigated through the monitoring of the changes in the $\mathrm{CA}$ of water droplets in the dark conditions for several months (Fig. 7). After the UV-light irradiation was ceased, high hydrophilicity was maintained for ten days

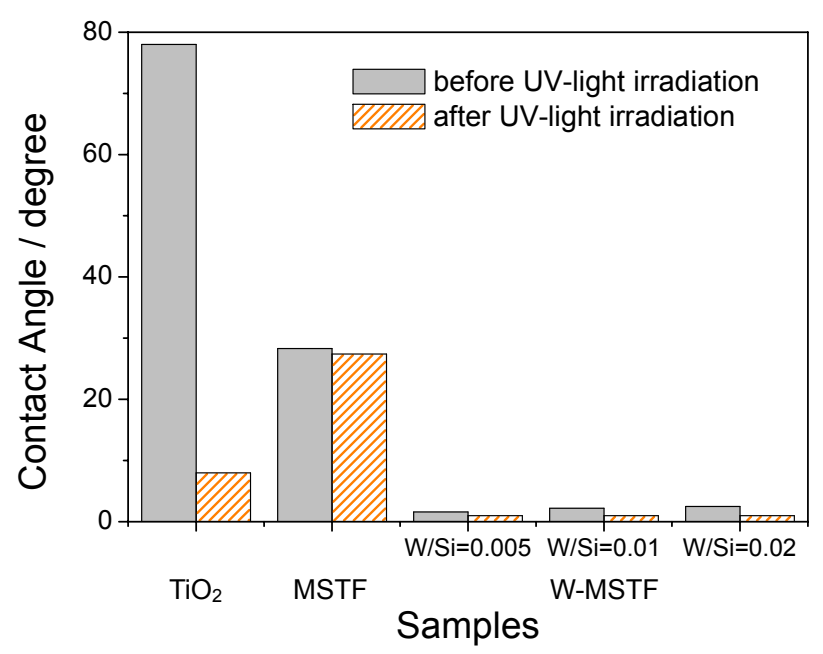

FIG. 5: Contact angle of the water droplets on $\mathrm{TiO}_{2}$ thin film, pure MSTF, and W-MSTFs with different tungsten concentration $(\mathrm{W} / \mathrm{Si}=0.005,0.01$, and 0.02$)$ before and after the UV-light irradiation for $2 \mathrm{~h}$.

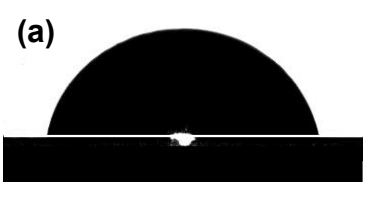

(b)

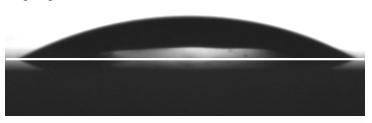

(c)

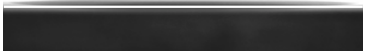

(A)

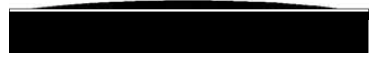

(B)

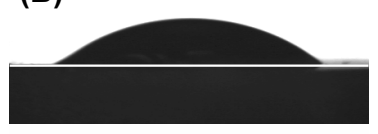

(C)

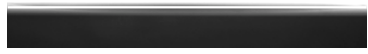

FIG. 6: Photographic images of the water droplets on $\mathrm{TiO}_{2}$ thin film (a, A), pure MSTF (b, B), and W-MSTFs (W/Si = $0.01)(\mathrm{c}, \mathrm{C})$ before $(\mathrm{a}-\mathrm{c})$ and after $(\mathrm{A}-\mathrm{C})$ the UV-light irradiation for $2 \mathrm{~h}$.

without significant increase of CA. After one month, the $\mathrm{CA}$ of water droplets was gradually increased and reached around $10^{\circ}$ by the adhesion of oily dusts in the air. However, highly hydrophilic surface of the W-MSTF was immediately recovered under the UV-light irradiation even after three months later. These results clearly indicated that W-MSTFs possessed high durability on the quartz plates and maintained hydrophilic surface with relatively low CA of water even in the dark for a long period, showing potential applications in the facile coating material for the functionalization of mirrors as well as window glasses.

\section{CONCLUSIONS}

Mesoporous silica thin films containing isolated tetrahedrally-coordinated tungsten oxide species (WMSTFs) were successfully prepared on the quartz plates 


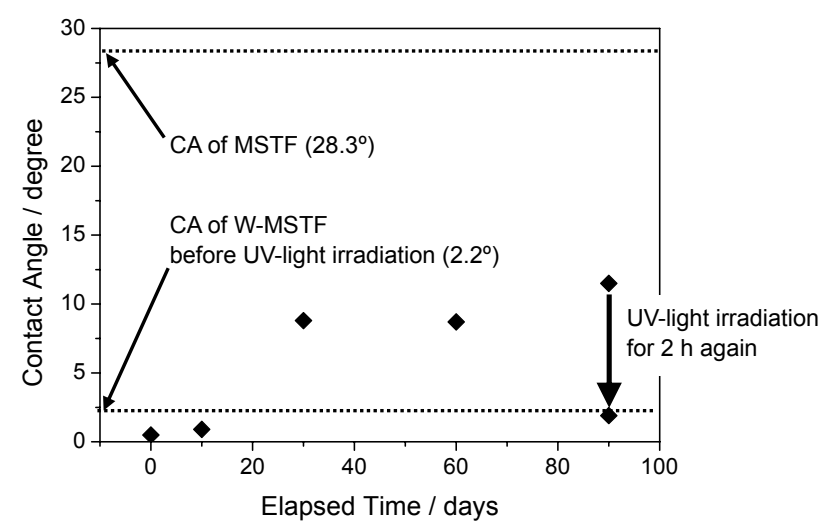

FIG. 7: Time-dependent contact angle measurement of WMSTF $(\mathrm{W} / \mathrm{Si}=0.01) \mathrm{kept}$ in dark conditions after the UVlight irradiation for $2 \mathrm{~h}$. using a sol-gel/spin coating method. The surface of these thin films exhibited the superior hydrophilic properties even before the UV-light irradiation. It was also found that W-MSTFs showed the photoinduced superhydrophilicity under the UV-light, although the CA of water on pure MSTFs was hardly changed even after prolonged UV-light irradiation. Therefore, isolated tetrahedrallycoordinated tungsten oxide species within the framework structures of MSTFs played significant roles for the enhancement of surface hydrophilic properties.

\section{Acknowledgments}

This study is supported by Grants-in-Aid for Scientific Research (KAKENHI) from Ministry of Education, Culture, Sports, Science and Technology.
[1] A. Corma, Chem. Rev. 97, 2373 (1997).

[2] K. Kageyama, J.-I. Tamazawa, and T. Aida, Science 285 , 2113 (1999).

[3] S. Jun, S. H. Joo, R. Ryoo, M. Kruk, M. Jaroniec, Z. Liu, T. Ohsuna, and O. Terasaki, J. Am. Chem. Soc. 122, 10712 (2000).

[4] P. Wu and T. Tatsumi, Catal. Suv. Asia 8, 137 (2004).

[5] H. Yamashita and M. Anpo, Curr. Opin. Solid State Mater. Sci. 7, 471 (2003).

[6] Y. Masui, S. Ohshiro, M. Anpo, T. Ohmichi, I. Katayama, and H. Yamashita, e-J. Surf. Sci. Nanotech. 3, 448 (2005).

[7] H. Yamashita and K. Mori, Chem. Lett. 36, 348 (2007).

[8] K. Mori, Y. Kondo, S. Morimoto, and H. Yamashita, J. Phys. Chem. C 112, 397 (2008).

[9] R. Wang, K. Hashimoto, A. Fujishima, M. Chikuni, E.
Kojima, A. Kitamura, M. Shimohigoshi, and T. Watanabe, Nature 388, 431 (1997).

[10] M. Ogawa, K. Ikeue, and M. Anpo, Chem. Mater. 13, 2900 (2001).

[11] N. Nishiyama, J. Kaihara, Y. Nishiyama, Y. Egashira, and K. Ueyama, Langmuir 23, 4746 (2007).

[12] Y. Horiuchi, K. Mori, N. Nishiyama, and H. Yamashita, Chem. Lett. 37, 748 (2008).

[13] H. Yamashita, S. Nishio, S. Imaoka, M. Shimada, K. Mori, T. Tanaka, and N. Nishiyama, Top. Catal. 47, 116 (2008).

[14] X.-L. Yang, W.-L. Dai, R. Gao, and K. Fan, J. Catal. 249, 278 (2007).

[15] L. Lizama and T. Klimova, Appl. Catal. B Environ. 82, 139 (2008). 